\title{
Restauration d'un faux moignon sous une couronne céramique existante
}

\section{Abutment restauration under an existing ceramic crown}

\section{MOTS-CLEFS :}

- Tenon en fibre, fracture du moignon, reconstitution corono-radiculaire

\section{KEYWORDS:}

- Fiber post, stump fracture, post and core restauration

\section{Résumé}

L'évolution des restaurations esthétiques et les exigences élevées des patients ont conduit à envisager la reconstitution corono-radiculaires également esthétiques. Plusieurs options peuvent alors être envisagées. Cependant, lorsqu'on est amener à restaurer un moignon sous une couronne céramique existante, la décision n'est pas évidente.

Ce travail détermine les conditions de cette réintervention, et décrit les étapes de restauration du moignon, ò l'aide des tenons en fibre, tout en conservant la couronne céramique.

\section{Abstract}

The evolution of esthetic restorations and the high claims of patients have led to take into account the use of post and core reconstructions that are also aesthetic. Several options can then be considered. However, when we are led to restore a stump beneath an existing ceramic crown, the decision is not obvious.

This work determines the conditions of this re-intervention, and describes the steps to restore the stump, using fiber posts, while preserving the ceramic crown.
AOS 2014;269:4-9

DOI: $10.1051 / \mathrm{aos} / 2014302$

(C) EDP Sciences 2014

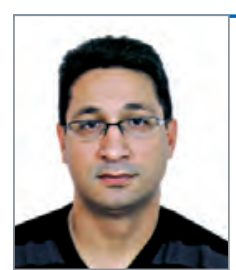

- Hicham SOUALHI, Professeur assistant en prothèse fixée. Faculté de médecine dentaire. Universite Mohamed V Souissi, Rabat Maroc

Ahd ZAGHBA, Professeur assistant en prothèse fixée.

Amal EL YAMANI, Professeur assistant en prothèse fixée.

Professeur agrégé de l'enseignement supérieur 


\section{INTRODUCTION}

La restauration des dents antérieures est souvent une tâche délicate, en raison de l'importance de ces dents dans la communication et l'esthétique. Les restaurations tout en céramique ont été mises au point pour imiter l'aspect des dents naturelles. Leur principal avantage, par opposition aux restaurations céramo-métalliques, est leur capacité à transmettre la lumière grâce à leur translucidité élevée [1].

Cependant, la réalisation de ces restaurations céramo-céramiques, dont la translucidité répond aux exigences esthétiques actuelles, oblige à se poser et à résoudre des problèmes spécifiques : le faux moignon, support coronaire de l'élément prothétique, ne doit pas être visible au travers de la couronne tout céramique. Le concept de reconstitution translucide dans un souci esthétique doit être anticipé au stade des reconstitutions corono-radiculaires (RCR) [2-4].

Les reconstitutions corono-radiculaires esthétiques en plus des exigences communes à toute RCR - de protéger et renforcer la dent en répartissant les contraintes fonctionnelles à l'ensemble des tissus de soutien et d'assurer la rétention du matériau de reconstitution destiné à remplacer la substance coronaire détruite $[5,6]-$ doivent répondre à d'autres spécificités permettant de rétablir ou d'améliorer l'esthétique $[2,4]$. Ainsi, elles ne doivent pas modifier la teinte de la couronne et elles doivent contribuer à éclaircir la région cervicale. En général, le résultat esthétique d'une restauration tout céramique va être influencé par :

$\checkmark$ la teinte du moignon;

$\checkmark$ la teinte de la restauration;

$\checkmark$ la teinte du matériau de collage.

La couleur du moignon après préparation doit être examinée attentivement. Il est essentiel de donner la teinte des tissus dentaires sous-jacents sur la fiche de laboratoire. Actuellement, il existe des teintiers qui permettent de relever la couleur du moignon, ce qui permet au céramiste d'adapter la teinte et la luminosité de la restauration en fonction du moignon sous-jacent. Pour les systèmes céramiques, plusieurs choix d'infrastructure permettent d'obtenir des aspects naturels en fonction des situations cliniques. Une infrastructure translucide est indiquée sur des piliers pulpés sans dyschromies, afin de permettre la diffusion de la lumière dans la dent et les tissus marginaux. A contrario, un pilier décoloré (dyschromie radiculaire, faux moignon métallique) nécessite une infrastructure plus opaque (zircone) afin de supprimer toute influence défavorable sur l'apparence naturelle.

S'il existe bien différentes techniques de reconstitutions corono-radiculaires esthétiques, aucune encore à ce jour ne peut être considérée comme la solution idéale.
Le choix est encore plus difficile lorsqu'on est amené à réaliser une reconstitution corono-radiculaire sous une couronne céramique existante.

Généralement, les techniques suivantes peuvent être utilisées pour restaurer le moignon :

$\checkmark$ technique indirecte sur un modèle en laboratoire : le patient est privé de la restauration pendant la fabrication de l'inlay-core en laboratoire ;

$\checkmark$ technique intrabuccale directe avec résine acrylique autopolymérisable : elle consiste au montage par le praticien directement en bouche de la maquette de l'inlay-core en résine calcinable. Cette maquette sera secondairement coulée au laboratoire.

Nous nous proposons de décrire une autre solution de restauration du moignon à l'aide de tenon en fibres tout en conservant la couronne céramique. À travers un cas clinique, nous allons illustrer les conditions et les étapes de cette réintervention.

\section{DIFFÉRENTS TYPES DE RECONSTITUTIONS CORONO-RADICULAIRES ESTHÉTIQUES}

Les reconstitutions corono-radiculaires esthétiques sont élaborées par différents procédés.

\section{Reconstitution indirecte}

\Reconstitutions métalliques céramisées : l'utilisation des métaux pour la réalisation des inlay-cores est justifiée par un très grand recul clinique et une simplicité de réalisation au laboratoire. L'aspect disgracieux du métal au niveau coronaire sera masqué par une couche d'opaque ou mieux par une couche opaque et une couche de céramique $[7,8]$.

\econstitutions indirectes en céramique : afin de supprimer totalement le métal, l'utilisation de reconstitution indirecte en céramique a été suggérée : en oxyde d'alumine infiltré de verre «Inceram » claveté avec un tenon en fibres, une technique alliant un tenon en zirconium $\mathrm{ZrO}_{2}$ sur lequel est pressé une céramique Empress ${ }^{\circledast}$, ou également une technique permettant d'obtenir un inlay-core entièrement en céramique obtenu par usinage (Celay) $[1,4,9]$.

$\checkmark$ Reconstitution indirecte en composite de laboratoire : l'évolution des composites de laboratoire (système Targis R, Vectris R, Ivoclar Vivadent) permet actuellement, en association avec des tenons en fibres, de réaliser des reconstitutions corono-radiculaires répondant parfaitement au cahier des charges [4]. 

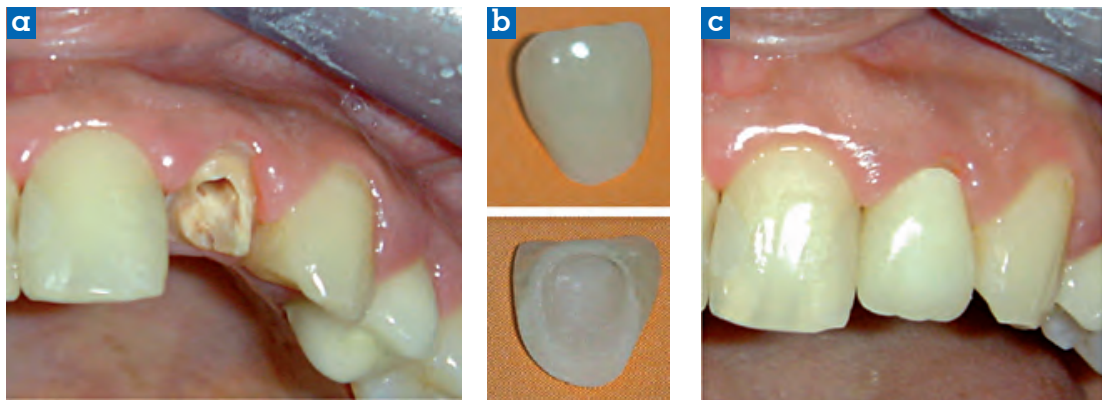

$<$ Fig. 1 :

a. Situation initiale : fracture du moignon.

b. Couronne céramique E-max.

c. Essayage de la couronne qui témoigne de sa bonne adaptation.

\section{Reconstitution directe}

I Reconstitution directe en clinique : Une reconstitution corono-radiculaire associant un tenon en fibres de verre ou de quartz et une résine composite de reconstitution. Le tenon et le composite de reconstitution coronaires sont collés aux tissus dentaires. Les tenons fibrés sont composés pour $60 \%$ à $66 \%$ de fibres longitudinales, parallèles entre elles, unidirectionnelles, liées par des résines d'époxy ou de polyester (RelyX Fiber Post ${ }^{\oplus}, 3 \mathrm{M}$ ESPE, FRC Postec ${ }^{\oplus}$, Ivoclar Vivadent, Dentinpost ${ }^{\oplus}$, Komet, FiberKleer ${ }^{\varpi}$ ). Leur module d'élasticité est semblable à celui de la dentine. Leur profil de surface lisse en fait des tenons passifs qui n'exercent pas de stress sur la dentine radiculaire [10]. En plus de leur intérêt esthétique lors de la réalisation des couronnes céramo-céramiques, les reconstitutions corono-radiculaires à tenon fibré permettent une économie tissulaire, préservant ainsi la solidité de la dent dépulpée, contrairement aux reconstitutions corono-radiculaires coulées indirectes qui nécessitent une mise de dépouille interne de la dent [11]. De plus, certaines études ont montré que le collage permet un renforcement des structures dentaires restantes [12-14] et une répartition des contraintes fonctionnelles à l'ensemble de l'organe dentaire, réduisant ainsi le risque de fractures radiculaires.

Une reconstitution corono-radiculaire à tenon fibré est une alternative intéressante à l'inlay-core métallique ou en céramique. Celui-ci reste toutefois indiqué dans le cas de fort délabrement coronaire, avec peu ou pas de dentine cervicale périphérique et des limites juxtaou intra-sulculaires.

\section{PRÉSENTATION DU CAS CLINIQUE}

Une patiente de 27 ans se présente en urgence au service de prothèse fixée. Elle se plaint du descellement de la couronne céramique sur la 22. À l'examen, le moignon présente une fracture coronaire, sans atteinte des marges cervicales de la préparation, ce qui explique la perte de rétention et le descellement de la couronne (fig. 1a). Le fragment fracturé de la dent 22, qui était fixé à la couronne, a été retiré de la face interne de la couronne. Il s'agit d'une couronne céramique E-max (fig. 1b) qui, bien que descellée, conserve toujours une bonne adaptation aux limites cervicales et assure une très bonne intégration biologique, fonctionnelle et esthétique (fig. 1c). La patiente rapporte que la couronne a été réalisée récemment par un confrère (6 mois).

L'examen radiologique met en évidence un traitement endodontique correct (fig. 2).

L'attitude la plus recommandée était la réalisation d'une nouvelle couronne céramique ainsi qu'une reconstitution corono-radiculaire esthétique. Cette option a été rejetée du fait que la couronne présentait toujours une bonne intégration occlusale, esthétique et fonctionnelle, et aussi pour des raisons financières. Afin de conserver la couronne céramique et pour restaurer le moignon, les techniques indirectes peuvent être proposées. Elles permettront de réaliser un inlaycore en métal « céramisé », ou en céramique ou en composite. Cependant le protocole de réalisation aussi bien en clinique qu'en laboratoire est fastidieux en plus d'un coût et d'un temps de réalisation importants. Ainsi :

$\checkmark$ l'inlay-core métallique céramisé ne permet pas de résoudre l'assombrissement cervical et radiculaire et présente des risques de corrosion ;

$\checkmark$ l'inlay-core en céramique Empress ou monobloc (usiné) nécessite d'utiliser un tenon en céramique, ce qui est incompatible avec la forme du système canalaire de l'incisive latérale. Par ailleurs, la rigidité de la céramique et surtout la dépose quasi impossible contre-indiquent cette technique ;

$\checkmark$ le système de l'inlay-core en céramique Inceram claveté par tenon en fibre semble séduisant, cependant le protocole d'élaboration sous une couronne existante est très compliqué et ne garantit pas à la fois une bonne adaptation de l'inlay-core sur la préparation et la conservation de la couronne. 


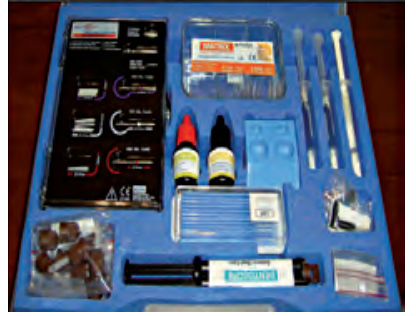

$<$ Fig. 3 :

Coffret de restauration corono-radicualire.
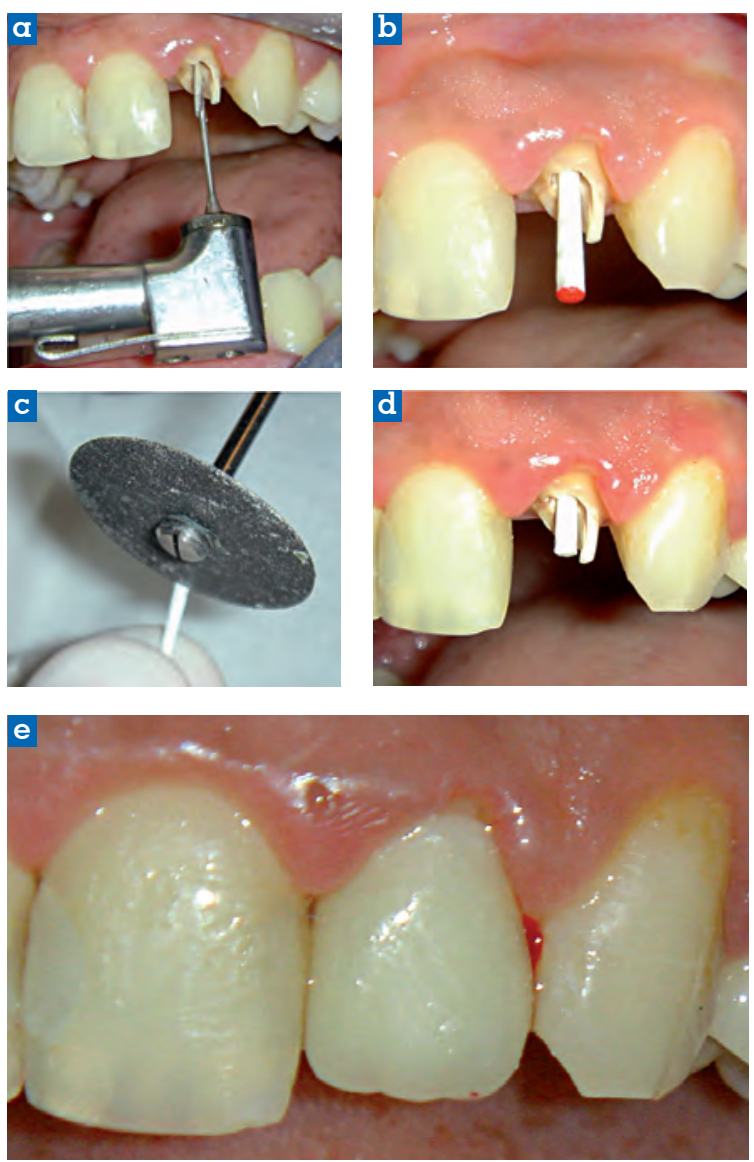

$\triangle$ Fig. 4 :

a. Forage canalaire, foret Gates et Largo.

b. Essayage du tenon.

c. Section du tenon à l'aide d'un disque.

d. Contrôle de la longueur du tenon.

e. Essayage de la couronne au-dessus du tenon.
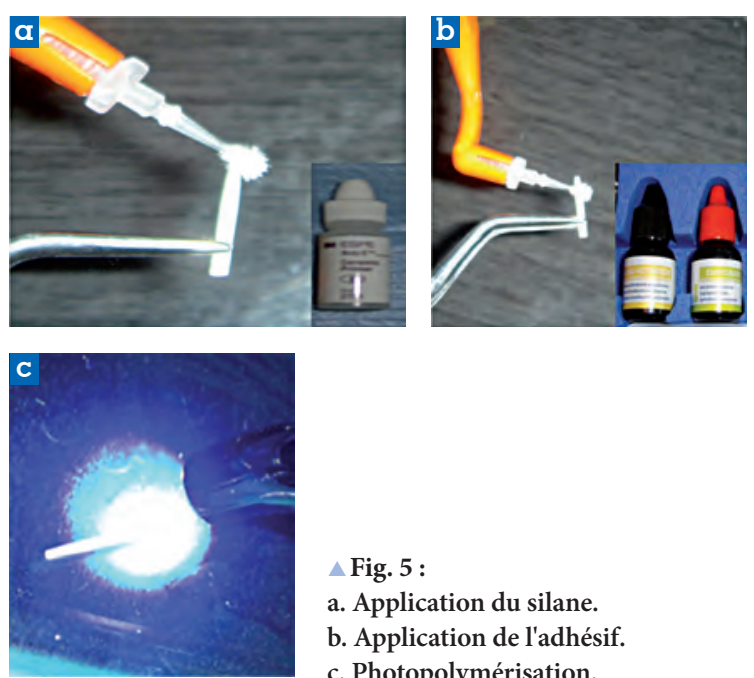

$\triangle$ Fig. 5 :

a. Application du silane.

b. Application de l'adhésif.

c. Photopolymérisation.
Une technique directe de reconstitution à l'aide de tenon en fibre est plus appropriée. Permettant de conserver l'intégration de la couronne céramique existante, avec une meilleure économie tissulaire, un protocole plus accessible, un coût moindre et surtout une réalisation en clinique en un seul temps répondant ainsi à l'urgence de la patiente.

On a opté pour un coffret de restauration corono-radiculaire aux tenons en fibres qui présente l'avantage que le composite de collage sert également à restaurer le moignon (fig. 3).

Le protocole opératoire de restauration se déroule en plusieurs étapes.

\section{Radiographie pré-opératoire.}

Elle nous renseigne sur la morphologie canalaire et la qualité du traitement endodontique et permet de déterminer la longueur du logement du tenon et son diamètre (fig. 2).

\section{Forage canalaire et adaptation du tenon en fibre.}

L'espace nécessaire à l'insertion du tenon en fibres a été réalisé en laissant un bouchon apical de $6 \mathrm{~mm}$, à l'aide de foret Gates et Largo, puis fini par des forets calibrés au tenon fibré. Ensuite le tenon est inséré dans le canal afin de contrôler sa bonne insertion et de déterminer la longueur de la partie intracoronaire de telle façon qu'il n'interfère pas avec l'intrados prothétique. Le tenon est sectionné à l'aide d'un disque diamanté (fig. 4 a-e).

\section{Le conditionnement du tenon}

et des tissus dentaires.

La surface du tenon est recouverte d'une couche de silane, laissée en attente 1 minute puis séchée. Ensuite, on procède à l'application de l'adhésif qui va être polymérisé (fig. 5 a-c).

Parallèlement, les tissus dentaires sont traités. Un mordançage est réalisé par un gel d'acide phosphorique à $37 \%$ pendant 30 secondes L'acide phosphorique est soigneusement rincé de façon à ne laisser aucune trace dans le système canalaire.

Le logement canalaire est ensuite séché avec des pointes de papier. C'est un séchage doux qui ne doit en aucun cas déshydrater la dentine. Puis une couche d'adhésif dual est appliquée à l'aide d'une brossette à l'intérieur du système canalaire (fig. 6 a-c).

Le composite de collage est injecté dans le logement canalaire à l'aide d'un embout spécifique puis le tenon est inséré. La photopolymérisation s'effectue en deux temps : photopolymérisation de 3 secondes pour gélifier la colle et permettre un retrait facile des excès qui ont débordé au-delà du canal, suivie d'une photopolymérisation de 40 secondes (fig. 7 a, b). 
Spécial Prothèse fixe Soualhi H., Zaghba A., El Ouali R., El Yamani A.
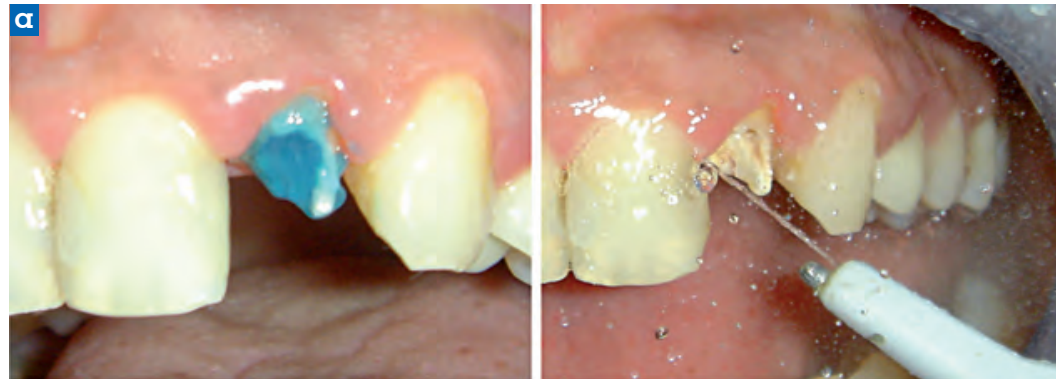

$\triangleleft$ Fig. 6 :

a. Mordançage à l'acide

orthophosphorique et rinçage.

b. Séchage.

c. Application de l'adhésif.
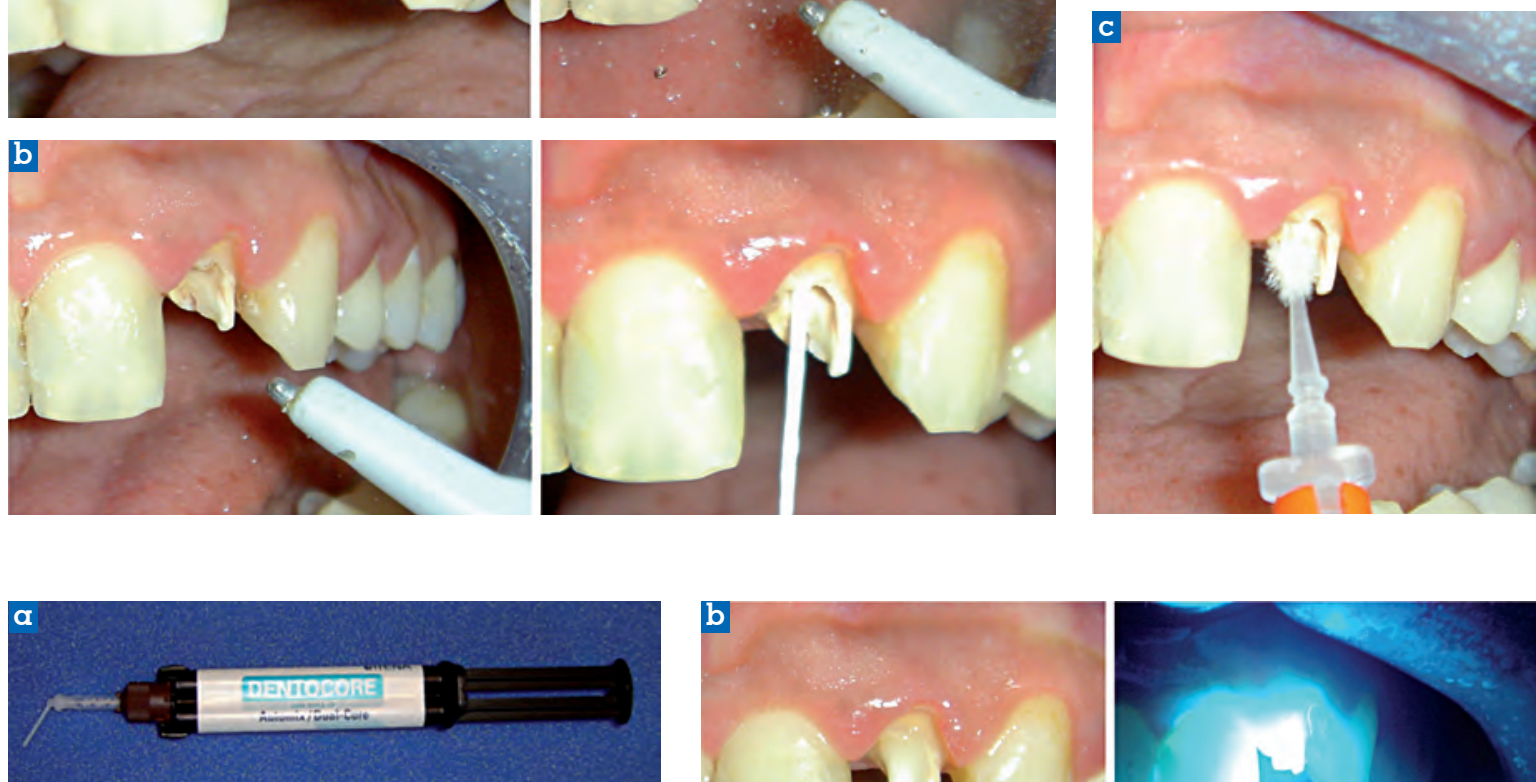

$\triangle$ Fig. 7 :

a. Composite de collage muni d'un embout spécifique.

b. Mise en place du composite et du tenon

et photopolymérisation.
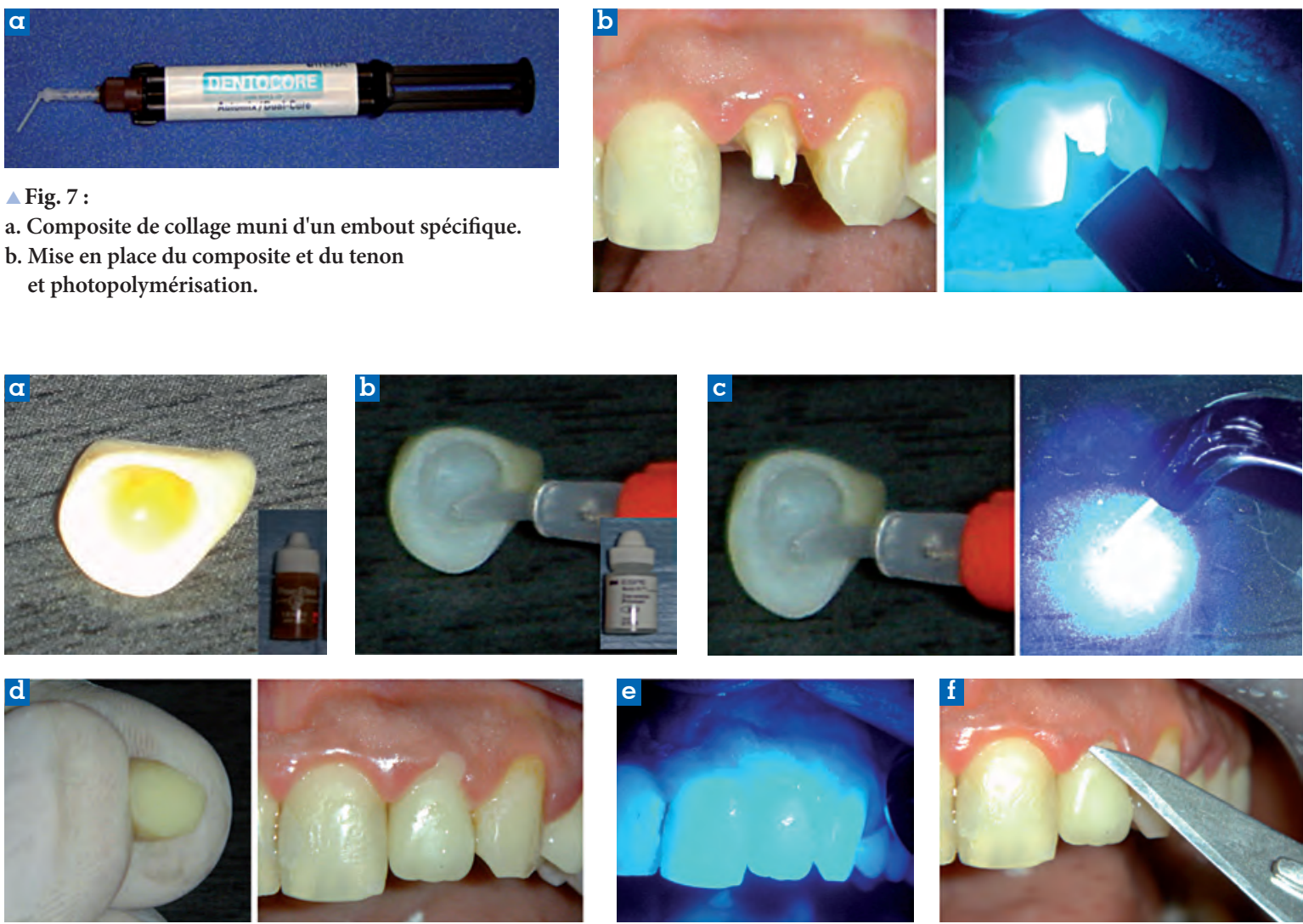

$\triangle$ Fig. 8 :

a. Mordançage à l'acide fluorhydrique.

b. Application du silane.

c. Application de l'adhésif et photopolymérisation. d. Mise en place du composite dans l'intrados de la couronne et insertion de la couronne sur la préparation au-dessus du tenon en fibre.

e. Photopolymérisation.

f. Élimination des excès.
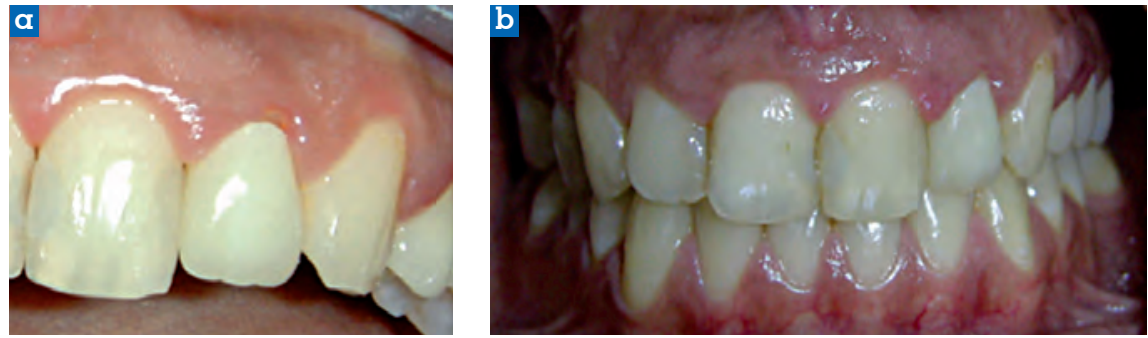

$\checkmark$ Fig. 9:

a. Résultat définitif.

b. Sourire de la patiente mettant en évidence l'intégration de la couronne. 


\section{Traitement de surface de la couronne céramo-céramique et restauration du moignon.}

L'intrados prothétique préalablement nettoyé sera mordancé par l'acide fluorhydrique puis rincé et séché. Une couche de silane sera appliquée et laissée à sécher 1 à 2 minutes. Ensuite, on applique une couche d'adhésif qui sera photopolymérisée, enfin le composite de reconstitution dual (qui est le même que celui du collage) est injecté à l'intérieur de la couronne qui sera par la suite repositionnée sur la préparation. Après photopolymérisation, les excès seront éliminés (fig. 8 a-f, fig. 9 a-b).

\section{CONCLUSION}

Devant toute fracture du moignon sous une couronne se pose le problème de la réintervention avec ou sans conservation de la couronne.

Dans le cas de conservation de la couronne céramique, le traitement sera d'autant plus difficile qu'il faut réaliser une reconstitution corono-radiculaire esthétique afin de profiter pleinement du caractère esthétique de la couronne céramique. Actuellement, seule une analyse clinique critique évaluant l'étiologie, les conditions d'intégration de couronne et tenant compte des différents procédés de réalisation des RCR esthétiques permettent de décider de maintien ou non de l'ancienne prothèse.

L'option de reconstitution corono-radiculaire à tenon fibré, grâce à l'amélioration de la performance des systèmes adhésifs, est aujourd'hui fiable et bénéficie d'un protocole reproductible. Elle doit donc faire partie de notre arsenal thérapeutique et les indications peuvent être étendues et proposées comme une alternative intéressante pour la restauration du moignon en conservant la couronne existante.

Il ne s'agit en aucun cas d'appliquer une thérapeutique unique à l'ensemble des situations cliniques, mais bien de choisir la solution technique la mieux adaptée au cas clinique en optimisant pour le patient le rapport bénéfice/coût.

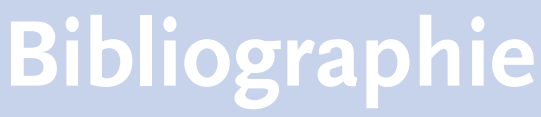

[1] Margossian P, Laborde G. Restaurations céramocéramiques. EMC 23-272-C-15, Elsevier Masson, 2007.

[2] Fradeani M, Aquilano A, Barducci G. Aestetic restoration of endodontically treated teeth. Pract Periodontal Aesthet Dent 1999;11(7):761-768.

[3] Freedman GA. Esthetic post-andcore treatment. Dent Clin North Am 2001; 45(1):103-116.

[4] Gonthier S, Desreumaux-Gothier M. Influence esthétique des reconstitutions corono-radiculaires. Réalités Cliniques, 2003;14(4):443-449.
[5] Sarfati E, Harter JC, Radiguet J. Évolution des conceptions des reconstitutions des dents dépulpées. Le tenon radiculaire : de son existence à sa disparition. Cah Prothèse 1995;90:71-78.

[6] Aboudharam G, Laurent M. Évolution et indications des reconstitutions coronoradiculaires indirectes. Cah Prothèse 2001;116: 61-70.

[7] Descamp F, Étienne O. Inlay-core, une technique encore d'actualité. Cah Prothèse 2010;150:59-68.

[8] Brousseaud J, Bartala M, Blanchard J-P, Faun S. Restaurations coronoradiculaires coulées (inlay-cores). Indications et procédures. Réalités Cliniques 2011;22(1):57-64.

[9] Descombes Y, Giordano D, Sieber C, Marinello C. Faux-moignons en céramique. Une nouvelle application des systèmes Celay $\%$ In-Ceram. Information Dentaire $\mathrm{n}^{\circ} 25$ du 23 juin 1999.
[10] Johnson ME, Stewart GP, Nielsen CJ, Hatton JF. Evaluation of root reinforcement of endodontically treated teeth. Pathol Oral Radiol Endod 2000;90(3):360-4.

[11] Dietschi D, Duc O, Krejci I, Sadan A. Biomechanical considerations for the restoration of endodontically treated teeth: a systematic review of the literature, part II (evaluation of fatigue behaviour, interfaces, and in vivo studies). Quintes Int 2008;39(2):117-129.

[12] Mendoza DB, Eaklew S, Kahl EA, Ho R. Root reinforcement with bonded preformed post. J Prosth Dent 1997;78:10-15.

[13] Newman MP, Yaman P, Dennison J, Rafter M. Fracture resistance of endodontically treated teeth restored with composite posts. $J$ Prosth Dent 2003;89:360-367.

[14] Silva NR, et al. Influence of different post design and composition on stress distribution in maxillary central incisor: finite element analysis. Indian J Dent Res 2009;20(2):153-158. Cat. 2. 